\title{
Aberrant intermediate filament and synaptophysin expression is a frequent event in malignant melanoma: an immunohistochemical study of 73 cases
}

\author{
Ryan C Romano, Jodi M Carter and Andrew L Folpe \\ Department of Laboratory Medicine and Pathology, Mayo Clinic, Rochester, MN, USA
}

\begin{abstract}
Malignant melanomas are known to express vimentin, among other intermediate filaments. Though anomalous keratin expression by malignant melanoma has been reported, its frequency is not well-established and this phenomenon is not well-known. We have seen in consultation a number of malignant melanomas with anomalous expression of keratin, other intermediate filaments, or synaptophysin, and therefore studied a large group of primary and metastatic melanomas to determine the frequency of these events. About 73 cases of malignant melanoma (22 primaries and 51 metastases) from 71 patients (51 male, 20 female; mean 59 years, range 17-87 years) were retrieved from our archives. Prior diagnoses were confirmed by re-review of hematoxylin and eosin sections and relevant (e.g., S100 protein, HMB45, Melan-A, and tyrosinase) immunohistochemical studies. Available sections were immunostained for keratin (OSCAR and AE1/AE3 antibodies), desmin, neurofilament protein, glial fibrillary acidic protein, synaptophysin, and chromogranin $A$. Not all cases could be tested for all markers. Cases were predominantly epithelioid $(48 / 73,66 \%)$ or spindle cell/desmoplastic (25/73, 34\%). S100 protein, Melan-A, HMB45, and tyrosinase were positive in 60/65 (92\%), 34/64 (53\%), 30/60 (50\%), 25/48 (52\%) of cases, respectively. All five S100-protein-negative cases expressed at least one of the other melanocytic markers: Melan-A (two of four, 50\%), HMB45 (two of three, 67\%), and tyrosinase (one of two, 50\%). All cases expressed at least one melanocytic marker. Cases were positive for keratin (OSCAR, 17/61, 28\%; AE1/AE3, $16 / 40,40 \%)$, desmin $(11 / 47,24 \%)$, neurofilament protein $(5 / 31,16 \%)$, glial fibrillary acidic protein $(3 / 32,9 \%)$, and synaptophysin $(10 / 34,29 \%)$, typically only in a minority of cells. Chromogranin was negative (0/32, $0 \%)$. Altogether $9 / 73$ cases $(12 \%)$ showed expression of $>1$ intermediate filament. All S100-protein-negative melanomas showed anomalous intermediate filament expression (keratin-one case, desmin-three cases, neurofilament protein-one case). Anomalous intermediate filament or synaptophysin expression was more common in epithelioid (intermediate filament, 27/48, 56\%; synaptophysin, 7/22, 32\%) as compared with spindle cell/desmoplastic (intermediate filament, 8/25, 32\%; synaptophysin, 3/12, 25\%) melanomas. Overall, 48\% (35/73) of cases showed anomalous expression of at least one intermediate filament. Anomalous expression of all intermediate filaments and synaptophysin was found in significant subsets of malignant melanoma, representing potentially serious diagnostic pitfalls. While the inclusion of consultation cases may inflate the frequency of these findings in this series, similar findings were also seen in institutional cases. Malignant melanoma showing anomalous intermediate filament and synaptophysin expression may easily be mistaken for carcinomas, rhabdomyosarcomas, and neuroendocrine tumors. Awareness of this phenomenon, careful histopathological evaluation, and an appropriate melanocytic immunohistochemical panel should facilitate the diagnosis of malignant melanoma with unusual immunophenotypes.
\end{abstract}

Modern Pathology (2015) 28, 1033-1042; doi:10.1038/modpathol.2015.62; published online 29 May 2015

Correspondence: Dr AL Folpe, MD, Department of Laboratory Medicine and Pathology, Mayo Clinic, 200 1st Street Southwest, Rochester, MN 55905, USA.

E-mail: Folpe.Andrew@Mayo.edu

Received 17 February 2015; accepted 2 April 2015; published online 29 May 2015
The histopathological diagnosis of malignant melanoma is typically straightforward, particularly for cases with an in situ component or pigmentation. However, many melanomas lack diagnostic histopathological features, and require ancillary 
immunohistochemical studies for markers such as S100 protein, HMB45, Melan-A, tyrosinase, microphthalmia transcription factor (MiTF), and SOX10 for definitive diagnosis. The notoriously protean appearance of melanoma often generates a broad, differential diagnosis, including a wide variety of other epithelioid, round cell, and spindle cell neoplasms. Thus, melanoma-associated immunohistochemical markers often comprise only one part of a larger immunohistochemical panel, including various intermediate filament proteins (e.g., keratins for carcinoma and desmin for myogenic tumors) and neuroendocrine markers (e.g., synaptophysin and chromogranin A), among others.

With widespread use of ancillary immunohistochemistry, and near-universal use of epitope retrieval techniques, pathologists have come to appreciate that many neoplasms may occasionally show unexpected expression of any number of markers, so-called 'anomalous' or 'aberrant' immunoreactivity. ${ }^{1}$ Welldescribed examples of this phenomenon include keratin expression in angiosarcoma, ${ }^{2}$ leiomyosarcoma, ${ }^{3}$ and Ewing sarcoma, ${ }^{4}$ and synaptophysin expression in alveolar rhabdomyosarcoma. ${ }^{5}$ It is less widely appreciated that melanomas too may show aberrant expression of various immunohistochemical markers, with a relatively small number of reports, of melanomas showing expression of intermediate filaments other than vimentin (e.g., keratins, desmin, neurofilament protein, and glial fibrillary acidic protein) ${ }^{6-8}$ and/or neuroendocrine markers. ${ }^{9-11}$

Over the past several years, we have seen in consultation a significant number of melanomas in which aberrant expression of intermediate filaments and/or neuroendocrine markers obscured the correct diagnosis, and prompted consideration of various non-melanocytic neoplasms, including carcinoma, rhabdomyosarcoma, neural neoplasms, and various neuroendocrine tumors. As no study to date appears to have comprehensively examined this issue, we evaluated a large number of well-characterized melanomas, using contemporary immunohistochemical techniques, to better determine the actual frequency of aberrant expression of these markers.

\section{Materials and methods}

This study was approved by the Mayo Clinic Institutional Review Board. All available slides and blocks from 73 cases of melanoma were retrieved from our institutional and consultation archives. Cases had been previously classified as 'melanoma' by a combination of clinical history, routine morphological evaluation, and ancillary immunohistochemistry for melanoma markers, including S100 protein, HMB45, Melan-A, and tyrosinase,. Antibodies to MiTF and SOX10 are not currently available in our clinical laboratory and were not utilized for diagnosis. For institutional cases, only definitive excision specimens were studied. For immunohistochemistry, 4- $\mu \mathrm{m}$ formalin-fixed, paraffinembedded tissue sections were immunostained using heat induced epitope retrieval, the Ventana Benchmark XT platform (Ventana Medical Systems, Tucson, AZ, USA), the Ultraview DAB detection system (Ventana), and commercially available antibodies to keratins (clone AE1/AE3, 1:200, Dako, Carpenteria, CA and clone OSCAR, 1:100, Covance, Princeton, NJ), desmin (clone DE-R-11, 1:100, Leica Biosystems, Newcastle Upon Tyne, United Kingdom), glial fibrillary acidic protein (polyclonal, 1:8000, Dako), neurofilament protein (clone 2F11, 1:2000 dilution, Dako), synaptophysin (clone 27G12, 1:50, Leica Biosystems), and chromogranin A (clone LK2H10, prediluted, Ventana Medical Systems). Not all cases could be tested for all markers, owing to insufficient available tissue. The immunohistochemical results were scored as 'negative' (no positive cells), ' $1+$ ' ( $<5 \%$ positive cells), ' $2+$ ' ( $6-25 \%$ positive cells), and ' $3+$ ' ( $>26 \%$ positive cells). Appropriate positive and negative controls were employed.

\section{Results}

The 73 melanomas included 22 primary lesions and 51 metastases from 71 patients (51 male, 20 female; mean age 59 years, range 17-87 years). Cases were classified as principally epithelioid (48 cases, 66\%) or spindle cell/desmoplastic (25 cases, 34\%) (Table 1). The immunohistochemical results are

Table 1 Immunohistochemical findings

\begin{tabular}{|c|c|c|c|}
\hline Antigen & Epithelioid melanoma & Spindle cell/desmoplastic melanoma & Total \\
\hline S100 protein & $39 / 43(91 \%)$ & $21 / 22(95 \%)$ & $60 / 65(92 \%)$ \\
\hline Melan-A & $31 / 44(70 \%)$ & $3 / 20(15 \%)$ & $34 / 64(53 \%)$ \\
\hline HMB45 & $29 / 41(71 \%)$ & $1 / 19(5 \%)$ & $30 / 60(50 \%)$ \\
\hline Tyrosinase & $19 / 28(68 \%)$ & $6 / 20(30 \%)$ & $25 / 48(52 \%)$ \\
\hline Keratin (AE1/AE3) & $13 / 29(45 \%)$ & $3 / 11(27 \%)$ & $16 / 40(40 \%)$ \\
\hline Keratin (OSCAR) & $15 / 38(39 \%)$ & $2 / 23(9 \%)$ & $17 / 61(28 \%)$ \\
\hline Desmin & $8 / 32(25 \%)$ & $3 / 15(20 \%)$ & $11 / 47(24 \%)$ \\
\hline Neurofilament protein & $4 / 21(19 \%)$ & $1 / 10(10 \%)$ & $5 / 31(16 \%)$ \\
\hline Glial fibrillary acidic protein & $2 / 23(9 \%)$ & $1 / 9(11 \%)$ & $3 / 32(9 \%)$ \\
\hline Synaptophysin & $7 / 22(32 \%)$ & $3 / 12(25 \%)$ & $10 / 34(29 \%)$ \\
\hline Any intermediate filament & $27 / 48(56 \%)$ & $8 / 25(32 \%)$ & $35 / 73(48 \%)$ \\
\hline
\end{tabular}


illustrated in Figures 1-6. Immunostains for S100 protein, Melan-A, HMB45, and tyrosinase were positive in 60/65 (92\%), 34/64 (53\%), 30/60 (50\%) and 25/48 (52\%) cases, respectively. The 5 S100protein-negative cases all expressed at least one of the other melanocytic markers.

Overall, $35 / 73(48 \%)$ of melanomas showed aberrant expression of at least 1 intermediate filament protein. This staining was typically confined to a small number of cells, with 28 cases $(74 \%)$ scored as ' $1+$ ', 5 tumors (13\%) scored as ' $2+$ ', and 5 lesions scored as ' $3+$ '. Aberrant keratin expression was the most common finding, present in 16/40 (40\%) cases with the AE1/AE3 cocktail and in 17/61 (28\%) cases with the OSCAR antibody. Desmin, neurofilament protein, and glial fibrillary acidic protein expression were less common, noted in 11/47 (24\%), 5/31 $(16 \%)$, and $3 / 32(9 \%)$ of tested cases, respectively. Additional testing at the time of initial diagnosis showed two of these desmin-positive melanomas to also express myogenin and MyoD1, indicative of rhabdomyoblastic differentiation. Nine of 73 cases $(12 \%)$ showed aberrant expression of keratins and another intermediate filament protein. Synaptophysin expression was seen in 10/34 (29\%) cases (1+, 8 cases; $2+, 1$ case, and $3+, 1$ case); none were chromogranin A positive (0/32).

Aberrant intermediate filament expression was more common in epithelioid melanomas (27/48 cases, $56 \%$ ), as compared with spindle cell/desmoplastic tumors $(8 / 25,32 \%)$. Similarly, anomalous synaptophysin expression was seen in 7/22 (32\%) of epithelioid melanomas, as compared with 3/12 (25\%) spindle cell/desmoplastic melanomas. No significant differences were seen in the percentages of primary versus metastatic melanomas showing aberrant intermediate filament and/or synaptophysin expression (primary tumors: 12/22, 55\%; metastatic tumors: 26/51, 51\%). Interestingly, all S100 protein-negative cases $(N)$ showed anomalous intermediate filament expression (desmin-3 cases; keratin and neurofilament protein-1 case each).

\section{Discussion}

The results of the present study suggest that aberrant expression of non-vimentin intermediate filaments
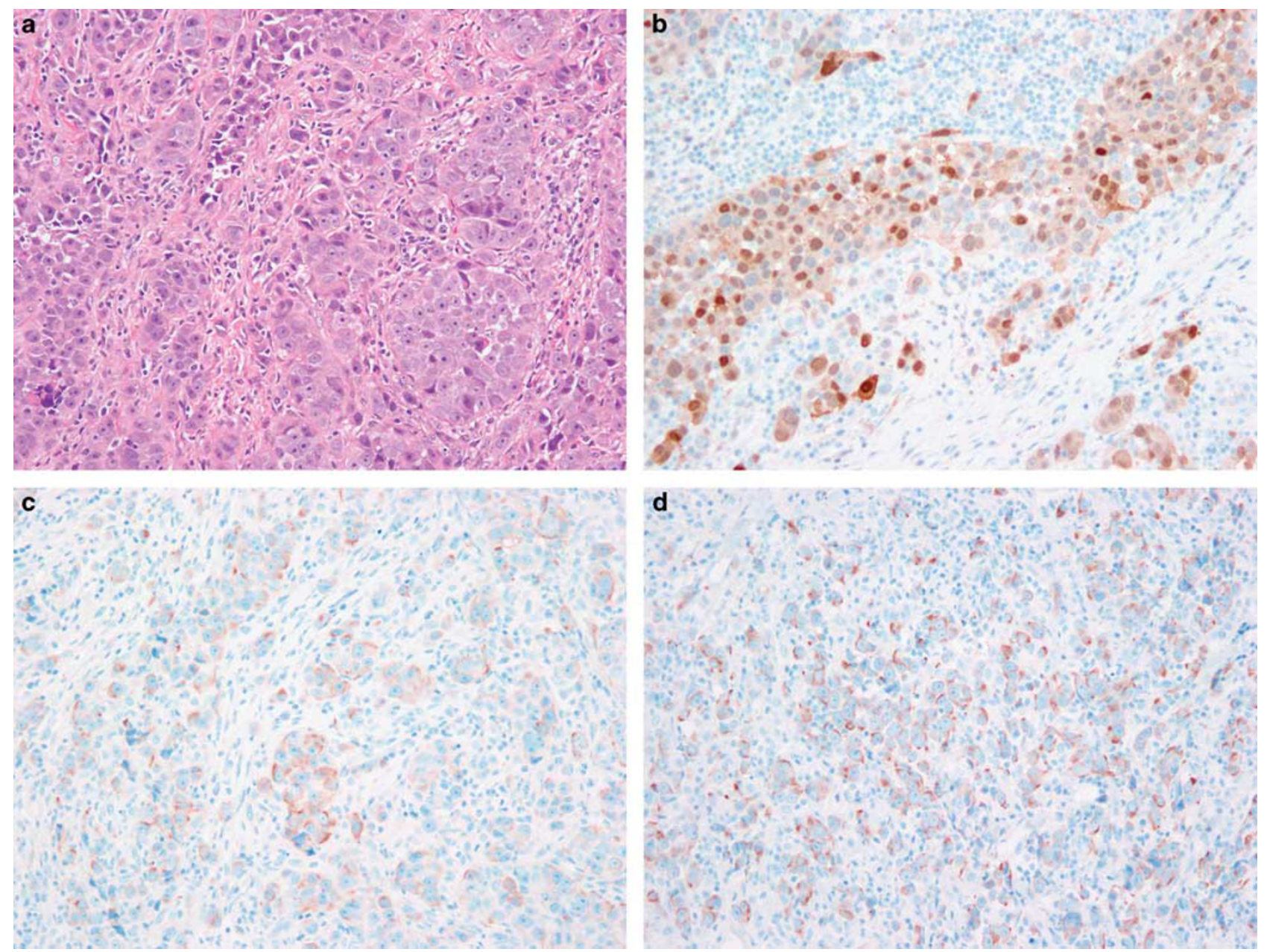

Figure 1 Primary epithelioid melanoma (a), positive for both S100 protein (b) and HMB45 (c). This case showed '2+' aberrant cytokeratin expression, using the AE1/AE3 antibody cocktail (d). 

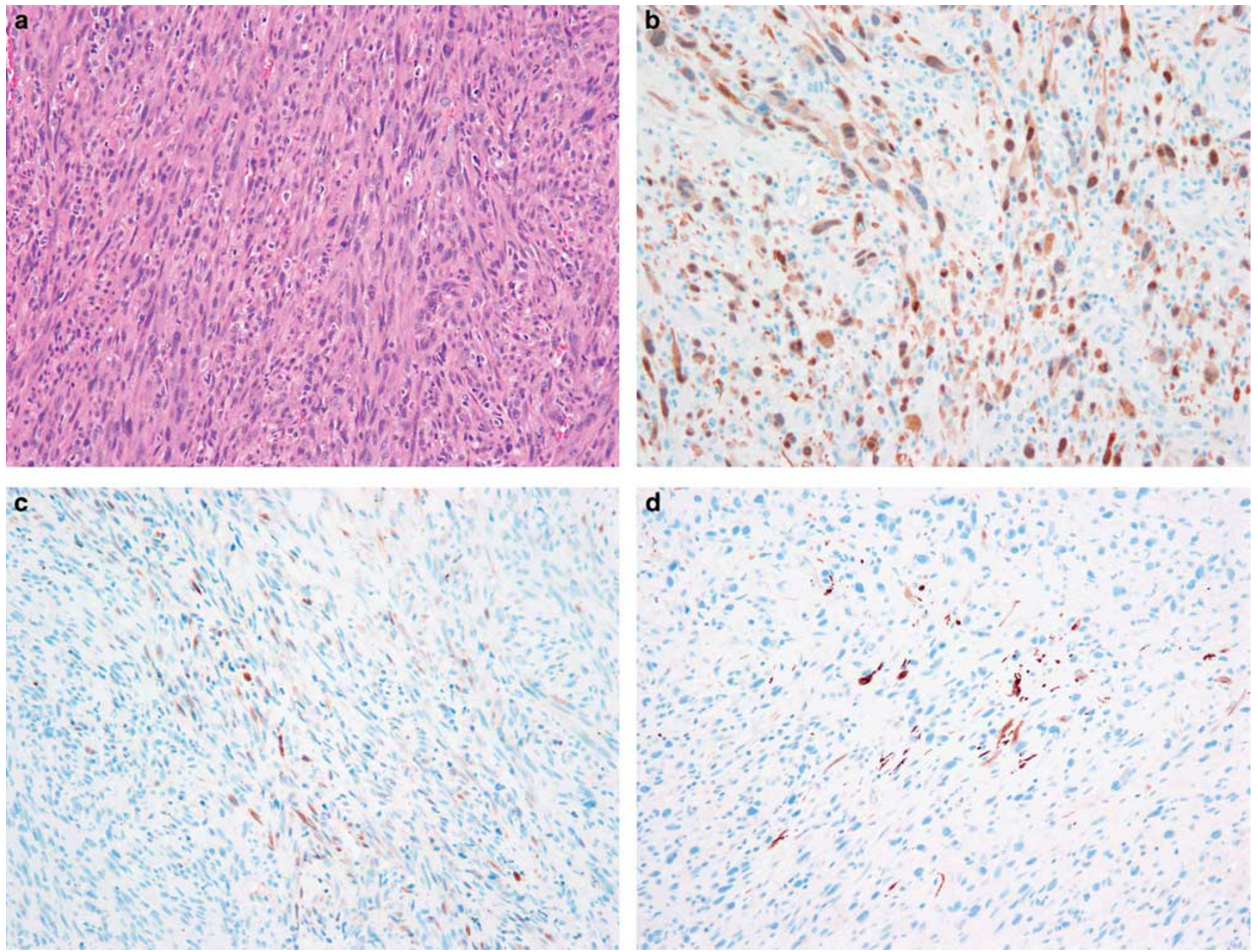

Figure 2 Primary spindle cell melanoma (a), strongly positive for S100 protein in a nuclear and cytoplasmic pattern (b). This case showed focal ('1+') aberrant expression of both cytokeratins, using the OSCAR antibody (c) and desmin (d).

and synaptophysin are relatively a common phenomena in melanoma, present in roughly equal numbers of primary melanomas and metastatic melanomas. While our results are in general agreement with the literature, the percentage of positive cases in the present series is somewhat higher than has previously been reported. This is likely due to selection bias secondary to our inclusion of consultation cases, at least some of which were referred to us specifically because of these findings.

The intermediate filaments, consisting of five principal subgroups (types I and II-the acidic and basic keratins; type III-desmin, glial fibrillary acidic protein, peripherin, and vimentin; type IV- $\alpha$-internexin, neurofilaments, synemin, and syncoilin; and type $\mathrm{V}$-nuclear lamins) comprise the major structural component of the cytoskeleton and have critical roles in the regulation of the nucleus and in the maintenance of cell stiffness, formation of lamellipodia, regulation of cell migration, and cell adhesion. ${ }^{12}$ With the advent of immunohistochemistry in the early 1980s, it was swiftly recognized that expression of intermediate filaments subgroups was restricted in human tissues and their neoplastic derivatives, with mesenchymal tissues and tumors chiefly expressing vimentin, epithelia and carcinomas showing keratin expression, and muscle of all types and myogenous tumors displaying desmin expression. ${ }^{13-15}$ The earliest immunohistochemical studies of melanoma, performed chiefly by immunofluorescence on fresh, frozen tissues, showed melanocytes and melanomas to express only vimentin. ${ }^{16-18}$

Gatter et $a l^{19}$ reported for the first time expression of keratins in melanoma, noting immunoreactivity in 4 of 41 fresh frozen melanomas with the commercially available Cam 5.2 antibody, and in up to 29 of 41 tumors with 1 of several proprietary antibodies (e. g., PK110 and PK121). These authors did not, however, find keratin expression in routinely processed melanomas with the Cam 5.2 antibody. These results were confirmed by Miettinen and Franssila, ${ }^{20}$ who showed Cam 5.2 immunoreactivity in a subset of frozen melanomas and in a smaller number of routinely processed tumors, and by Zarbo et al, ${ }^{6}$ who demonstrated keratin expression (using a variety of antibodies) in $21 \%, 8 \%$ and $2 \%$ of formalin-fixed, methacarn-fixed, and frozen melanomas, respectively. 

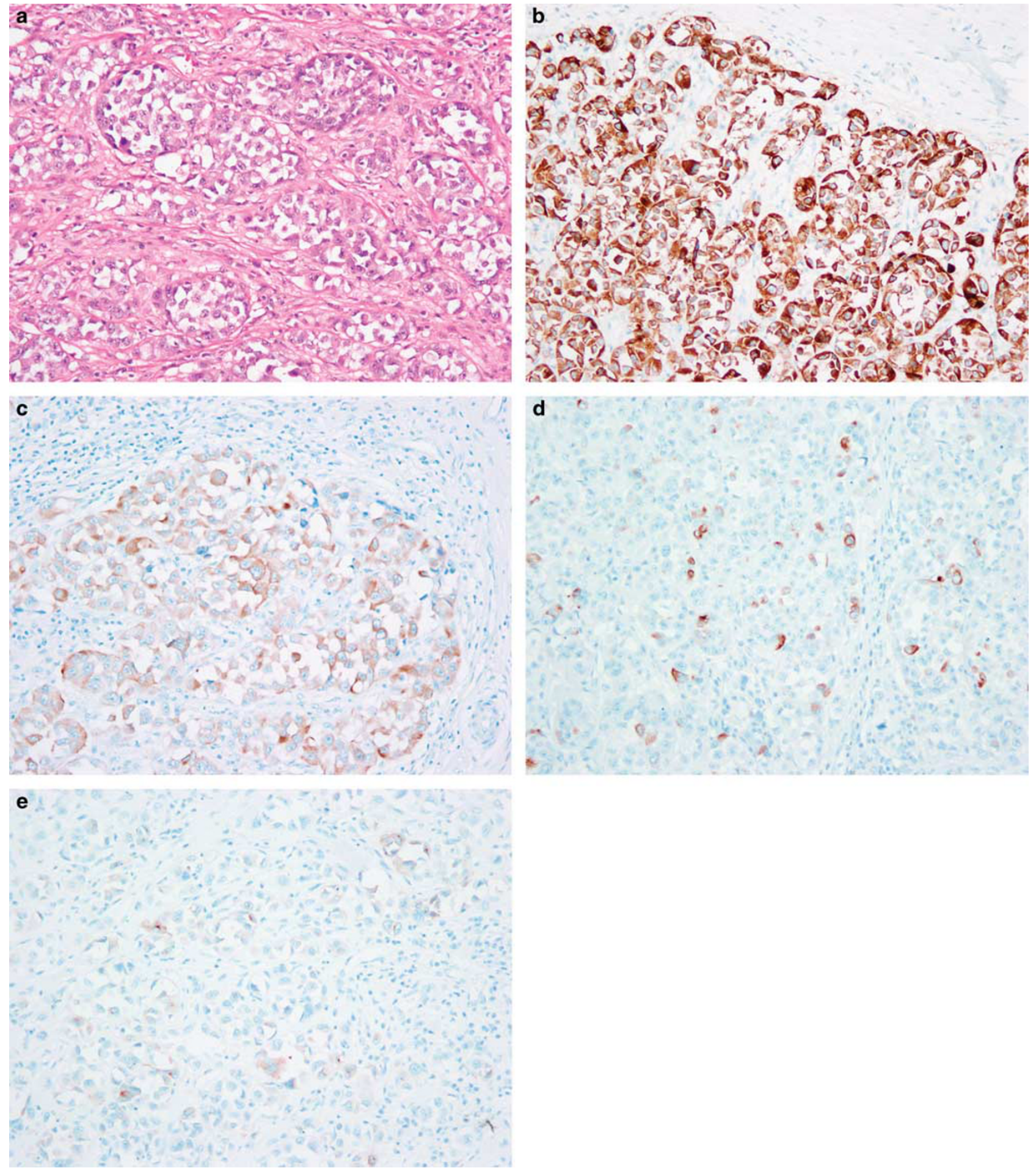

Figure 3 Primary epithelioid malignant melanoma (a), strongly positive for Melan-A (b). This case showed '2+' aberrant expression of cytokeratins (c), and was positive at '1+' with antibodies to neurofilament protein (d) and synaptophysin (e).

These investigators also demonstrated with western blot that keratin-immunoreactive melanomas were truly producing keratin peptides. Interestingly, Zarbo et $a l^{6}$ were only able to find aberrant keratin expression in recurrent or metastatic melanomas. Similar findings were reported by Ben-Izhak et $a l,{ }^{21}$ with aberrant keratin expression noted only in metastatic, as opposed to primary, melanomas. Subsequently, a modest number of studies have confirmed these early observations, with aberrant keratin expression reported in up to $10 \%$ of formalin-fixed, paraffin-embedded melanomas. ${ }^{7,22-25}$ Keratin mRNA expression in melanoma has also been confirmed with in situ hybridization. ${ }^{8}$ 

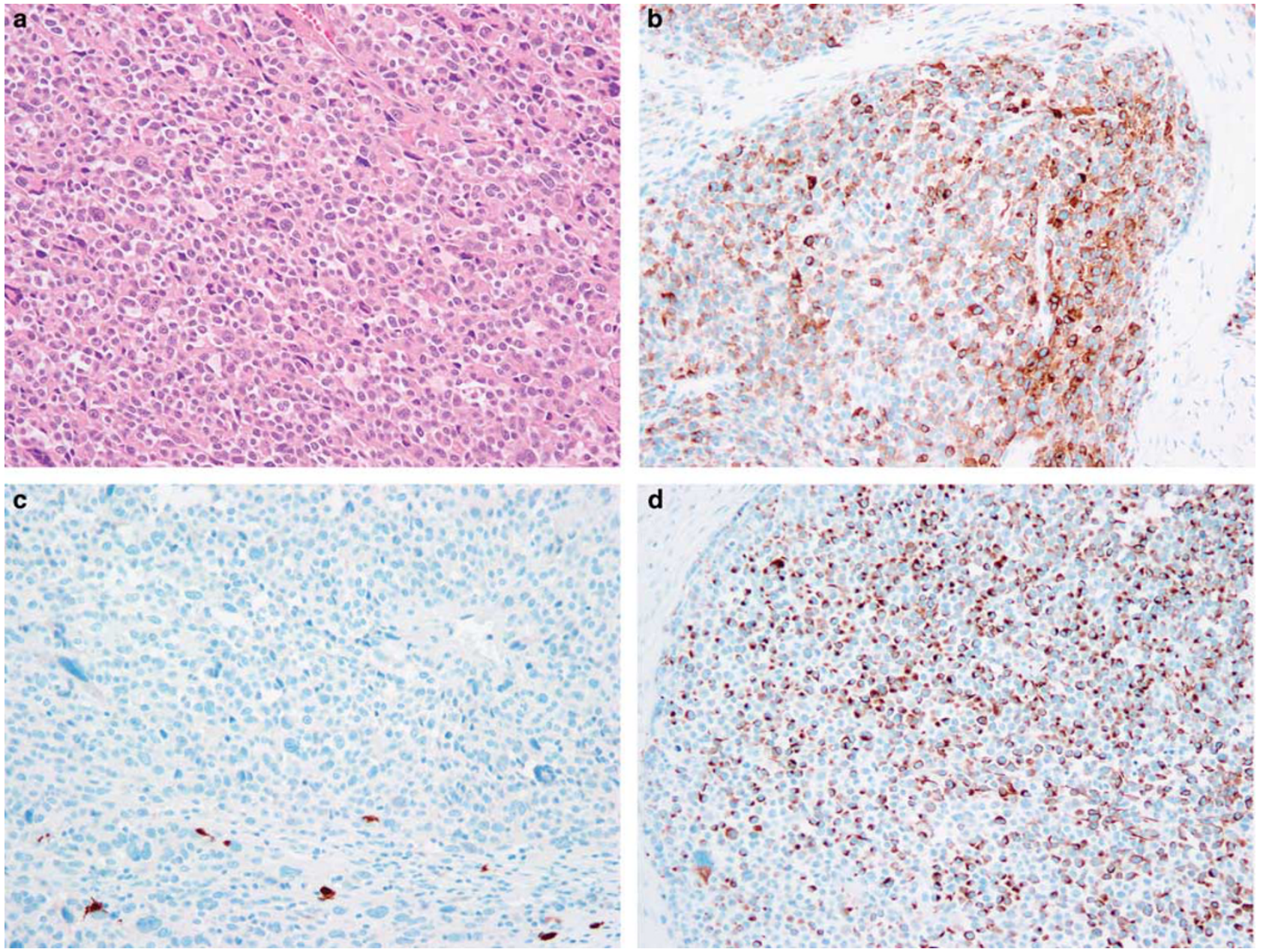

Figure 4 Metastatic melanoma, presenting in an axillary lymph node (a). This case was strongly Melan-A positive (b), but entirely S100 protein negative (c). Strong aberrant cytokeratin expression was seen (d).

Our present findings are in general agreement with the literature, with aberrant keratin expression noted in $40 \%$ of cases immunostained with the AE1/AE3 antibody cocktail, and $28 \%$ of cases immunostained with the OSCAR antibody. The higher frequency of keratin expression in the present series presumably reflects our use of modern epitope retrieval and detection techniques, as well as consultation bias. The lower sensitivity of the OSCAR antibody is most likely due to its identification of fewer keratins as compared with AE1/AE3. Although it is not known exactly which keratins OSCAR identifies, our own anecdotal experience shows the immunoreactivity of OSCAR to roughly parallel that of Cam 5.2 (which identifies keratins 8, 18, and 19). In contrast, AE1/ AE3 identifies a much broader range of acidic (AE1) and basic (AE3) keratins. In contrast to prior reports, however, we did not note any difference in the frequency of keratin expression in primary versus metastatic melanomas. Aberrant keratin expression was, however, considerably more common in epithelioid, as compared with spindle cell/desmoplastic, melanomas.
As with keratins, the earliest immunohistochemical studies of desmin expression showed it to be restricted to smooth, skeletal, and cardiac muscle, and to tumors showing smooth or skeletal muscle differentiation. ${ }^{13-15}$ Similarly, the first studies specifically examining melanomas found them to be desmin-negative. ${ }^{20,26}$ Truong et $a 1^{27}$ were the first to note limited desmin expression in a single case of melanoma, studied as part of a much larger survey of desmin expression in human neoplasia. Subsequently, a very small number of desmin-positive melanomas have been reported, ${ }^{28-30}$ including some showing smooth ${ }^{31}$ or skeletal muscle differentiation. ${ }^{32}$ We are aware of only a single prior study, published in abstract form, which has examined melanomas more systematically for desmin expression. ${ }^{23}$ Surprisingly, we found desmin expression in $24 \%$ of cases, typically confined to only a small number of cells, but on occasion showing more diffuse labeling. Two of these 11 desmin-positive cases showed in addition morphological features suggestive of skeletal muscle differentiation, and were found to be positive for the skeletal muscle-specific 

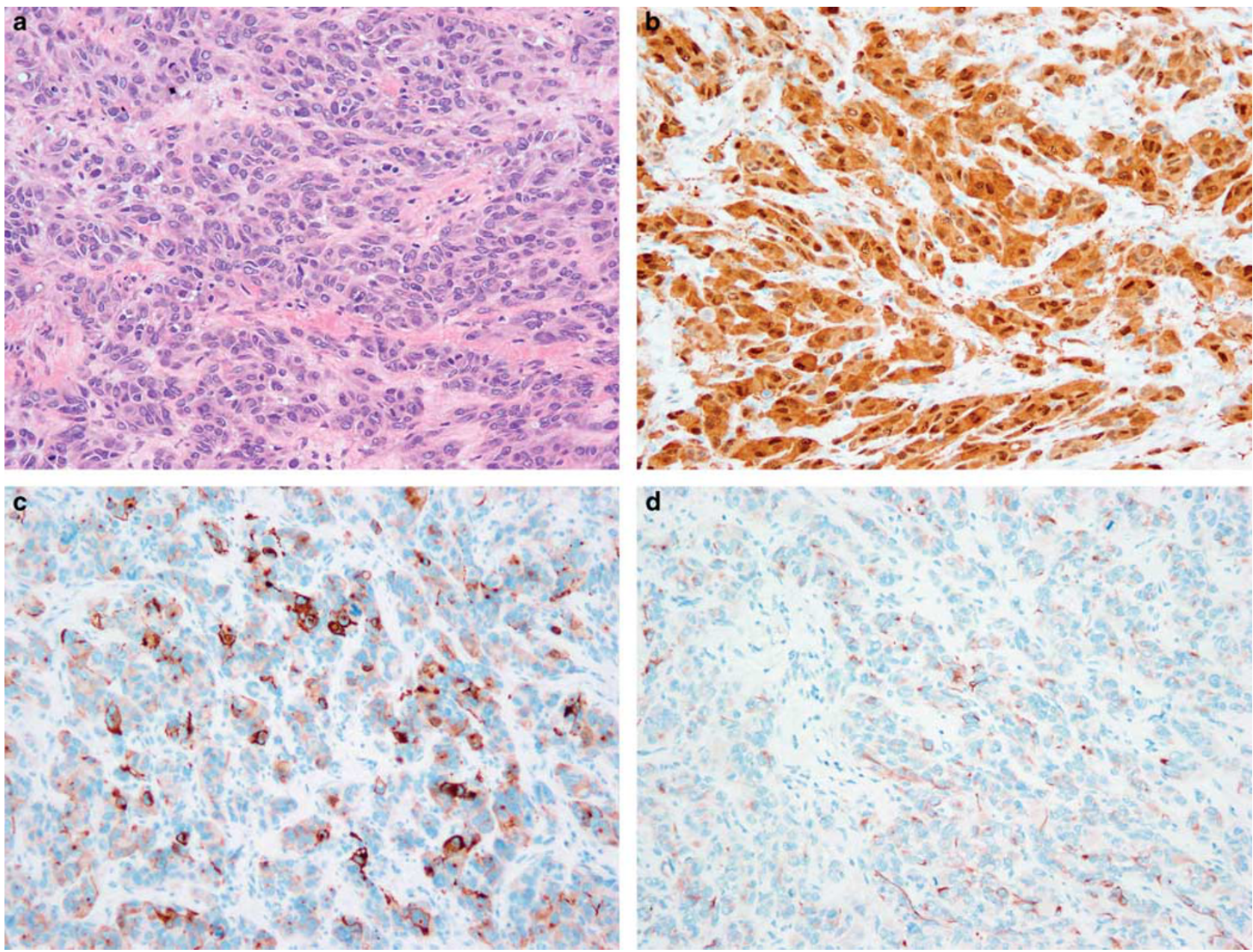

Figure 5 Melanoma, metastastic to the small bowel (a). This case was strongly positive for S100 protein (b) and HMB45 (not shown), and in addition showed strong expression of synaptophysin (c) and glial fibrillary acidic protein (d).

markers myogenin and MyoD1. Interestingly, aberrant desmin expression was a relatively frequent finding in S100 protein-negative melanomas, present in three of five such cases. This likely represents referral bias, as these were all consultation cases.

Aberrant expression of neurofilament protein and glial fibrillary acidic protein has also only very rarely been reported. Using frozen sections, Miettinen and Franssila, ${ }^{20}$ noted scattered neurofilament-positive cells in 2 of 15 metastatic melanomas, all of which were glial fibrillary acidic protein negative. Similarly, Shah et $a l^{33}$ found only a single neurofilamentpositive melanoma out of 64 examined melanomas. Neurofilament expression has, however, been reported in a higher percentage of melanomas in some small series, including those of Eyden et al, ${ }^{11}$ Lee et $a l^{9}{ }^{9}$ and the previously mentioned abstract published by Azam et al. ${ }^{23}$ Neurofilament expression has also been reported in very rare melanomas showing overt ganglioneuromatous differentiation. ${ }^{34}$ With the exception of the abstract published by Azam et al, ${ }^{23}$ we are aware of only a single study that has systematically evaluated glial fibrillary acidic protein expression in melanoma, by Iwamoto et al,,$^{35}$ showing expression in 9/17 (53\%) spindled and 2/10 (20\%) melanomas. We noted aberrant neurofilament protein and glial fibrillary acidic protein expression in 16\% and $9 \%$ of tested cases, respectively, confirming these prior observations.

Synaptophysin, a transmembrane glycoprotein present on presynaptic vesicles, is involved in the packaging, storage, and release of neurotransmitters and also functions as a membrane channel protein. ${ }^{36}$ Synaptophysin is normally expressed by neurons, endocrine and neuroendocrine cells, adrenal cortical cells and adrenocortical tumors, neuroendocrine carcinomas of various grades including Merkel cell carcinomas, paragangliomas, neuroblastomas, and esthesioneuroblastomas. ${ }^{37,38}$ Chromogranin A, a calcium-binding granin protein, is present in the dense core granules of neural and neuroendocrine cells. ${ }^{37,38}$ Chromogranin $\mathrm{A}$ expression in normal tissues generally parallels that of synaptophysin, although it is not expressed by adrenal cortical cells. $^{37,38}$ Aberrant synaptophysin expression is generally quite unusual, but may be seen in a consi- 

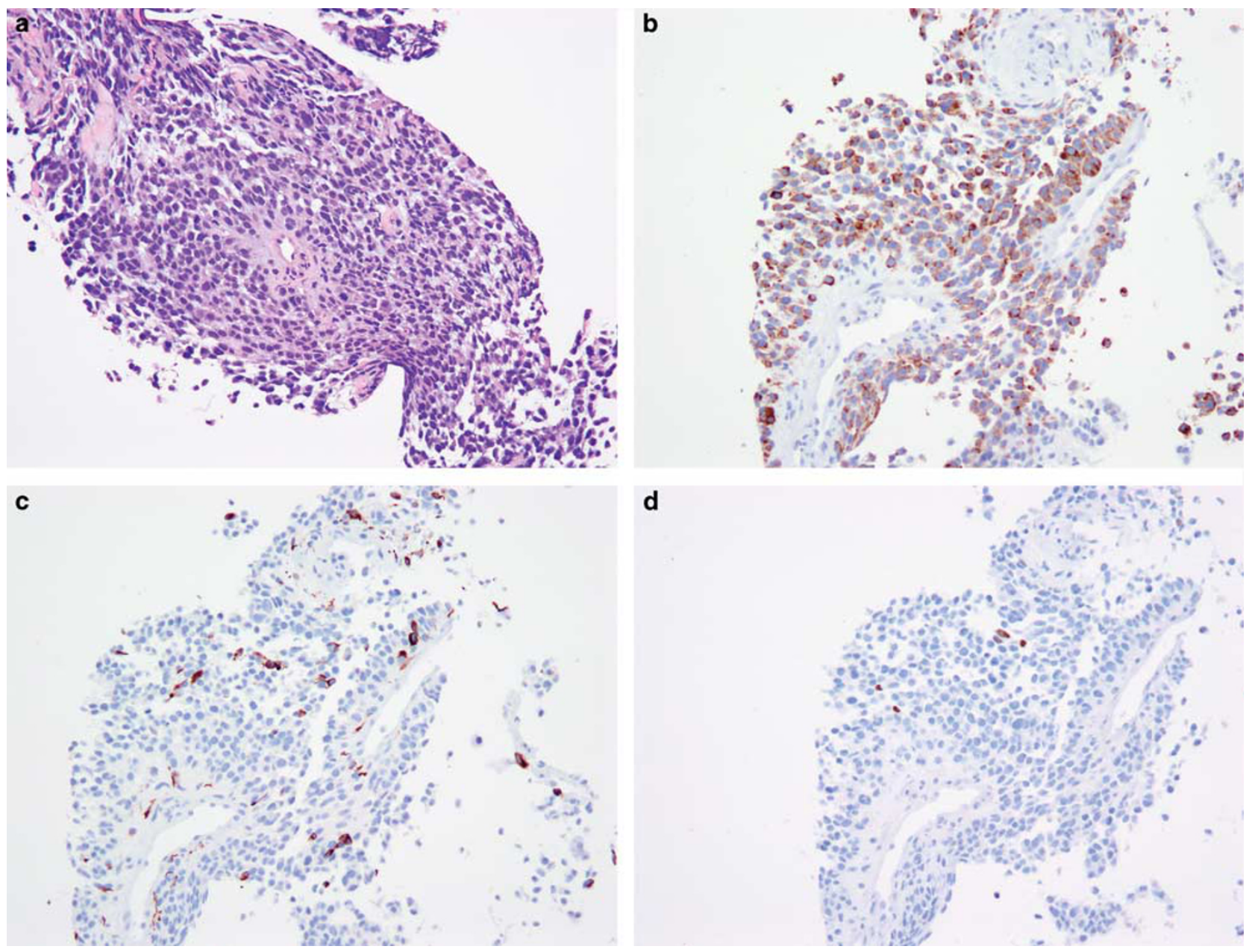

Figure 6 Metastatic melanoma, identified on a needle biopsy of a groin mass (a). In addition to showing Melan-A expression (b), this tumor was positive for desmin (c). Myogenin was also focally positive in a nuclear pattern, confirming heterologous rhabdomyosarcomatous differentiation.

derable percentage of alveolar rhabdomyosarcomas, ${ }^{5}$ a minority of extraskeletal myxoid chondrosarcomas, ${ }^{39}$ and very rare angiosarcomas. ${ }^{40}$ Aberrant chromogranin A exception is exceptional, seen only in very rare alveolar rhabdomyosarcomas ${ }^{5}$ and angiosarcomas. ${ }^{40}$ Synaptophysin expression has been reported previously in $<10$ melanomas, $, 9,10,34,41$ including 1 case with overt ganglioneuromatous differentiation ${ }^{34}$ and 2 cases showing actual neuroendocrine differentiation, in the form of chromogranin A co-expression and ultrastructurally confirmed dense core granules. ${ }^{41}$ We identified synaptophysin expression in nearly $30 \%$ of tested melanomas, suggesting that this phenomenon may be considerably more common than has been previously appreciated. In contrast, chromogranin A expression was absent in all cases.

The chief significance of aberrant intermediate filament and/or synaptophysin expression in melanoma is likely its potential for diagnostic confusion. Whether or not these findings become problematic is, however, highly idiosyncratic and dependent to a large degree on the clinical and morphological features of any given case, on the differential diagnosis generated by the reviewing pathologist, and on his/her immunohistochemical approach to the case. Obviously, aberrant marker expression will never be an issue for melanomas in which diagnosis is possible without ancillary immunostains or when only confirmatory immunostains for S100 protein and specific melanocytic markers are needed. Similarly, if the reviewing pathologist is aware of the potential for melanomas to show aberrant intermediate filament and/or synaptophysin expression, these findings should be the cause for little concern in lesions evaluated with a sufficiently broad immunohistochemical panel, including $\$ 100$ protein and other melanocytic markers. It is critical both that this panel not be limited to S100 protein (as roughly $2 \%$ of melanomas lack S100 protein expression) and that S100 protein not be omitted, as appreciable subsets of melanomas lack expression of HMB45, Melan-A, or tyrosinase..$^{42,43}$ More recently developed markers such as MiTF and/or SOX10 may also be of value in selected cases, although these markers lack perfect specificity for melanoma. ${ }^{44-48}$ In our experience, two other scenarios where aberrant marker 
expression in melanoma causes difficulty is when the referring pathologist hasn't considered the possibility of melanoma or is simply unaware that this may happen. Failing to include melanoma in the differential diagnosis seems most often to happen for lesions occurring in non-cutaneous locations (especially the sinonasal region and viscera), in the metastatic setting when a prior history of melanoma has not been provided, and in exclusively spindled or round cell (small cell) melanomas. The presence of diffuse, strong S100 protein immunoreactivity in a malignant-appearing spindle cell lesion should always prompt consideration of melanoma, especially as many such lesions will lack expression of other melanocytic markers. ${ }^{49}$ On the other hand, round cell melanomas, particularly in the sinonasal region, often show patchy or no $\$ 100$ protein expression, showing instead uniform expression of more specific melanocytic markers. ${ }^{9,50}$

In summary, we have identified aberrant expression of various intermediate filaments, including keratins, desmin, neurofilament protein and glial fibrillary acidic protein, and of the neuroendocrine marker synaptophysin in significant subsets of both epithelioid and spindle melanomas. These findings have obvious implications with regard to the use of ancillary immunohistochemical studies in the diagnosis of melanoma, and emphasize the need to employ a broad panel of markers in the differential diagnosis of poorly differentiated cutaneous tumors and in the setting of metastasis. Aberrant intermediate filament or synaptophysin expression in melanoma is typically confined to a minority of cells, an important clue. Thankfully, the overwhelming majority of melanomas showing aberrant expression of these proteins will express one (or more) of the specific markers of melanocytic differentiation (e.g., HMB45, Melan-A, and tyrosinase). Application of such markers is particularly critical for the correct diagnosis of S100 protein-negative melanomas, which seem to show aberrant intermediate filament protein expression in a higher than expected percentage of cases. Aberrant intermediate filament or synaptophysin expression in melanoma does not appear to be clinically significant, as it is seen in roughly similar percentages of primary tumors and metastases. Awareness by pathologists of these potentially significant pitfalls should greatly reduce the potential for misdiagnosis of these unusual tumors as non-melanocytic tumors.

\section{Disclosure/conflict of interest}

The authors declare no conflict of interest.

\section{References}

1 Swanson PE. HIERanarchy: the state of the art in immunohistochemistry. Am J Clin Pathol 1997;107: 139-140.
2 Gray $\mathrm{MH}$, Rosenberg AE, Dickersin GR et al. Cytokeratin expression in epithelioid vascular neoplasms. Hum Pathol 1990;21:212-217.

3 Gown AM, Boyd HC, Chang Y et al. Smooth muscle cells can express cytokeratins of 'simple' epithelium. Immunocytochemical and biochemical studies in vitro and in vivo. Am J Pathol 1988;132:223-232.

$4 \mathrm{Gu} \mathrm{M}$, Antonescu CR, Guiter G et al. Cytokeratin immunoreactivity in Ewing's sarcoma: prevalence in 50 cases confirmed by molecular diagnostic studies. Am J Surg Pathol 2000;24:410-416.

5 Bahrami A, Gown AM, Baird GS et al. Aberrant expression of epithelial and neuroendocrine markers in alveolar rhabdomyosarcoma: a potentially serious diagnostic pitfall. Mod Pathol 2008;21:795-806.

6 Zarbo RJ, Gown AM, Nagle RB et al. Anomalous cytokeratin expression in malignant melanoma: oneand two-dimensional western blot analysis and immunohistochemical survey of 100 melanomas. Mod Pathol 1990;3:494-501.

7 Fuchs U, Kivela T, Summanen $\mathrm{P}$ et al. An immunohistochemical and prognostic analysis of cytokeratin expression in malignant uveal melanoma. Am J Pathol 1992;141:169-181.

8 Chen N, Gong J, Chen X et al. Cytokeratin expression in malignant melanoma: potential application of in-situ hybridization analysis of mRNA. Melanoma Res 2009;19:87-93.

9 Lee H, Torres FX, McLean SA et al. Immunophenotypic heterogeneity of primary sinonasal melanoma with aberrant expression of neuroendocrine markers and calponin. Appl Immunohistochem Mol Morphol 2011;19:48-53.

10 Coli A, Giacomini PG, Bigotti G et al. Aberrant neurofilament protein and synaptophysin expression in malignant melanoma of the nasal cavity. Histopathology 2004;44:193-195.

11 Eyden B, Moss J, Shore I et al. Metastatic small cell malignant melanoma: a case requiring immunoelectronmicroscopy for the demonstration of lattice-deficient melanosomes. Ultrastruct Pathol 2005;29:71-78.

12 Gruenbaum Y, Aebi U. Intermediate filaments: a dynamic network that controls cell mechanics. F1000Prime Rep 2014;6:54.

13 Damjanov I. Antibodies to intermediate filaments and histogenesis. Lab Invest 1982;47:215-217.

14 Denk H, Krepler R, Artlieb U et al. Proteins of intermediate filaments. An immunohistochemical and biochemical approach to the classification of soft tissue tumors. Am J Pathol 1983;110:193-208.

15 Osborn M, Weber K. Tumor diagnosis by intermediate filament typing: a novel tool for surgical pathology. Lab Invest 1983;48:372-394.

16 Miettinen M, Lehto VP, Virtanen I. Presence of fibroblasttype intermediate filaments (vimentin) and absence of neurofilaments in pigmented nevi and malignant melanomas. J Cutan Pathol 1983;10:188-192.

17 Ramaekers FC, Puts JJ, Moesker O et al. Intermediate filaments in malignant melanomas. Identification and use as marker in surgical pathology. J Clin Invest 1983;71:635-643.

18 Caselitz J, Janner M, Breitbart E et al. Malignant melanomas contain only the vimentin type of intermediate filaments. Virchows Arch A Pathol Anat Histopathol 1983;400:43-51.

19 Gatter KC, Ralfkiaer E, Skinner J et al. An immunocytochemical study of malignant melanoma and its 
differential diagnosis from other malignant tumours. J Clin Pathol 1985;38:1353-1357.

20 Miettinen M, Franssila K. Immunohistochemical spectrum of malignant melanoma. The common presence of keratins. Lab Invest 1989;61:623-628.

21 Ben-Izhak O, Stark P, Levy R et al. Epithelial markers in malignant melanoma. A study of primary lesions and their metastases. Am J Dermatopathol 1994;16: 241-246.

22 Banerjee SS, Harris M. Morphological and immunophenotypic variations in malignant melanoma. Histopathology 2000;36:387-402.

23 Azam M, Bacchi C, Gown AM et al. Anomalous expresion of intermediate filament proteins: desmin, neurofilament and GFAP in melanomas. Mod Pathol 1999;12:55.

24 Korabiowska M, Fischer G, Steinacker A et al. Cytokeratin positivity in paraffin-embedded malignant melanomas: comparative study of KL1, A4 and Lu5 antibodies. Anticancer Res 2004;24:3203-3207.

25 Achilles E, Schroder S. Positive cytokeratin results in malignant melanoma. Pitfall in differential immunohistologic diagnosis of occult neoplasms. Pathologe 1994;15:235-241.

26 Leader M, Collins M, Patel J et al. Desmin: its value as a marker of muscle derived tumours using a commercial antibody. Virchows Arch A Pathol Anat Histopathol 1987;411:345-349.

27 Truong LD, Rangdaeng S, Cagle P et al. The diagnostic utility of desmin. A study of 584 cases and review of the literature. Am J Clin Pathol 1990;93:305-314.

28 Bittesini L, Dei Tos AP, Fletcher CD. Metastatic malignant melanoma showing a rhabdoid phenotype: further evidence of a non-specific histological pattern. Histopathology 1992;20:167-170.

29 Smith SM, Schmitt AC, Carrau RL et al. Primary sinonasal mucosal melanoma with aberrant diffuse and strong desmin reactivity: a potential diagnostic pitfall!. Head Neck Pathol 2015;9:165-171.

30 Pasz-Walczak G, Jesionek-Kupnicka D, Kubiak R et al. Rhabdomyosarcomatous (myoblastic?) phenotype of metastatic malignant melanoma. A case report. Pol J Pathol 2002;53:97-100.

31 Banerjee SS, Bishop PW, Nicholson CM et al. Malignant melanoma showing smooth muscle differentiation. J Clin Pathol 1996;49:950-951.

32 Gharpuray-Pandit D, Coyne J, Eyden B et al. Rhabdomyoblastic differentiation in malignant melanoma in adults: report of 2 cases. Int J Surg Pathol 2007;15: 20-25.

33 Shah IA, Gani OS, Wheler L. Comparative immunoreactivity of CD-68 and HMB-45 in malignant melanoma, neural tumors and nevi. Pathol Res Pract 1997;193:497-502.

34 Banerjee SS, Menasce LP, Eyden BP et al. Malignant melanoma showing ganglioneuroblastic differentiation: report of a unique case. Am J Surg Pathol 1999;23: $582-588$.
35 Iwamoto S, Burrows RC, Agoff SN et al. The p75 neurotrophin receptor, relative to other Schwann cell and melanoma markers, is abundantly Expressed in spindled melanomas. Am J Dermatopathol 2001;23: 288-294.

36 Gould VE, Wiedenmann B, Lee I et al. Synaptophysin expression in neuroendocrine neoplasms as determined by immunocytochemistry. Am J Pathol 1987;126:243-257.

37 Erickson LA, Lloyd RV. Practical markers used in the diagnosis of endocrine tumors. Adv Anat Pathol 2004;11:175-189.

38 Lloyd RV. Practical markers used in the diagnosis of neuroendocrine tumors. Endocr Pathol 2003;14:293-301.

39 Goh YW, Spagnolo DV, Platten $\mathrm{M}$ et al. Extraskeletal myxoid chondrosarcoma: a light microscopic, immunohistochemical, ultrastructural and immunoultrastructural study indicating neuroendocrine differentiation. Histopathology 2001;39:514-524.

40 Tessier Cloutier B, Costa FD, Tazelaar HD et al. Aberrant expression of neuroendocrine markers in angiosarcoma: a potential diagnostic pitfall. Hum Pathol 2014;45:1618-1624.

41 Eyden B, Pandit D, Banerjee SS. Malignant melanoma with neuroendocrine differentiation: clinical, histological, immunohistochemical and ultrastructural features of three cases. Histopathology 2005;47:402-409.

42 Bacchi CE, Bonetti F, Pea M et al. HMB-45. A review. Appl Immunohistochem 1996;4:73-85.

43 Busam KJ, Jungbluth AA. Melan-A, a new melanocytic differentiation marker. Adv Anat Pathol 1999;6: 12-18.

44 Pusiol T, Morichetti D, Zorzi MG et al. HMB-45 negative clear cell perivascular epithelioid cell tumor of the skin. Acta Dermatovenerol Croat 2012;20: 27-29.

45 Fox MD, Billings SD, Gleason BC et al. Expression of MiTF may be helpful in differentiating cellular neurothekeoma from plexiform fibrohistiocytic tumor (histiocytoid predominant) in a partial biopsy specimen. Am J Dermatopathol 2012;34:157-160.

46 Page RN, King R, Mihm MC Jr et al. Microphthalmia transcription factor and NKI/C3 expression in cellular neurothekeoma. Mod Pathol 2004;17:230-234.

47 Karamchandani JR, Nielsen TO, van de Rijn M et al. Sox10 and S100 in the diagnosis of soft-tissue neoplasms. Appl Immunohistochem Mol Morphol 2012;32:1291-1298.

48 Nonaka D, Chiriboga L, Rubin BP. Sox10: a panschwannian and melanocytic marker. Am J Surg Pathol 2008;32:1291-1298.

49 Longacre TA, Egbert BM, Rouse RV. Desmoplastic and spindle-cell malignant melanoma. An immunohistochemical study. Am J Surg Pathol 1996;20:1489-1500.

50 Regauer S, Anderhuber W, Richtig E et al. Primary mucosal melanomas of the nasal cavity and paranasal sinuses. A clinicopathological analysis of 14 cases. Apmis 1998;106:403-410. 